\title{
Polaronic Effects of a Hydrogenic Donor Impurity in Low Dimensional Semiconductor Systems
}

\author{
N. Radhakrishnan ${ }^{1}$ and A. J. Peter ${ }^{2}$ \\ ${ }^{1}$ Department of Biotechnology, IIT Madras, Chennai-600036, India \\ ${ }^{2}$ Department of Physics, Govt. Arts College, Melur-625106, India
}

Received 22 May 2009, accepted in revised form 24 July 2009

\begin{abstract}
The ground state of a polaron bound to hydrogen like donor impurity is investigated by considering the effect of bulk Longitudinal-Optical (LO) phonon. Donor binding energy of a hydrogenic donor, with the inclusion of electron-phonon interaction is computed for the low-dimensional semiconducting systems like quantum well, quantum well wire and quantum dot taking $\mathrm{GaAs} / \mathrm{Al}_{\mathrm{x}} \mathrm{Ga}_{1-\mathrm{x}} \mathrm{As}$ systems as an example. Calculations are performed using a variational approach within the single band effective mass approximation. The results show that the polaronic effect is more pronounced for the lowest confinement. The polaronic effect enhances the donor binding energy but it diminishes when the well width, wire radius and dot radius become larger. Also the numerical calculations reveal that the influences of phonons on donor are considerable and should not be neglected especially for narrow dimensions in all the three confinements.
\end{abstract}

Keywords: Donor binding energy; Polaronic effect; Quantum dot; Quantum wire; Quantum well.

C을 2009 JSR Publications. ISSN: 2070-0237 (Print); 2070-0245 (Online). All rights reserved.

DOI: $10.3329 /$ jsr.v1i3.2529

J. Sci. Res. 1 (3), $422-429$ (2009)

\section{Introduction}

With the advance of the modern molecular beam epitaxial and due to the recent progress in nanotechnology, it possible to fabricate low-dimensional hetero-structures, such as quantum wells (QWs) quantum well wires (QWWs) and quantum dots (QDs). They have been studied widely both theoretically and experimentally due to their potential application [1-3] especially in microelectronic device technology. In all the studies, it has been found out that the binding energy increases when going from bulk to QWs and from QWWs to QDs due to their reduction in confinements.

There is a great deal of work devoted to the study of the electron-phonon interactions in QWs $[4,5]$. It is well known that the electron-optical phonon interaction plays an

\footnotetext{
${ }^{2}$ Corresponding author: a.john.peter@gmail.com
} 
important part in the physical properties of polar crystals, especially in low-dimensional quantum systems [6] and it is believed that the electrons-longitudinal phonons are in the strong coupling regime leading to polaronic effect [7]. Guo and Chen presented the polaron effects on the second-harmonic generation in quantum well with an electric field [8]. The effect of the bulk Longitudinal-Optical (LO) phonon on the binding energy is investigated for a shallow donor impurity in a superlattice in the effective mass approximation by using the variational approach recently by Tayebi et al. [9] . They have obtained the results as a function of parameters which characterize the superlattice and the position of the impurity center.

Many authors $[10,11]$ found that the binding energy and polaronic effect of an exciton are greatly larger in QWW than in QW and predicted application to electronic and optoelectronic devices [12]. Bouhassoune et al. [13] have presented a study of the exciton binding energy in a cylindrical QWW in the presence of a uniform magnetic field by taking into account the interaction between the electron-hole and the two modes of phonons (confined LO and SO). The binding energies in QWWs have been found to reach values in the range of 2.5-3.5 times the bulk Rydberg.

Recent experimental studies have explored some novel physical phenomena such as quantum dot charging [14], transport through quantum dots [15, 16] and far infrared absorption [17] with potential electronic device applications. Numerical results for the low-lying spectra of parabolic quantum dots in which two electrons interact with each other through both coulomb repulsion and longitudinal-optical phonon under a magnetic field is presented recently [18]. The comparison of polaron effects in different nanostructures have shown that the maximum polaron effect is in the quantum dot [19].

Some recent optical measurements $[13,20]$ of the photoluminescence (PL) spectra realized on different quantum dots and quantum-well semiconductor structures, also reveal the LO phonon's effect on the PL line widths. Since the carrier charge-phonon interactions are essential to understand the experimental observation of the optical absorption spectra in semiconductors [21] the polaronic effect becomes a main subject of investigations in physics of low dimensional systems. All the research results have already shown that the electron-phonon interaction becomes more and more important in electronic properties and optical properties with the decreasing of the dimension.

In the present paper, we investigate the ground state of a polaron bound to a hydrogenic donor impurity by considering the effect of bulk Longitudinal-Optical phonon. The effect of electron-phonon interaction on the donor binding energy is employed for the low-dimensional semiconducting systems taking $\mathrm{GaAs} / \mathrm{Al}_{\mathrm{x}} \mathrm{Ga}_{1-\mathrm{x}} \mathrm{As}$ systems as an example. Calculations are performed by means of a variational approach within the effective mass approximation. In section 2, we briefly describe the method and the quantum well, wire and dot model used in our calculation. And the results and discussion are presented in section 3. A brief summary and results are presented in the last section. 


\section{Model and Calculations}

The Hamiltonian for a hydrogenic donor immersed in a dielectric medium of constant $\varepsilon$ with mass $\mathrm{m}^{*}$

$$
H=H_{e}+H_{p h}+H_{\text {int }}
$$

where $\mathrm{H}_{\mathrm{e}}$ describes the hydrogenic impurity term given by

$$
H_{e}=\frac{p^{2}}{2 m^{*}}-\frac{e^{2}}{\varepsilon r}+V
$$

$H_{p h}$ is the LO phonon Hamiltonian, $H_{\text {int }}$ is the electron-phonon interaction term, the expressions are taken from the Ref. [9] and V is the well potential confining the electron, such that

$$
\begin{aligned}
& V(z)=\left\{\begin{array}{cc}
0 & |z| \leq \frac{L}{2} \\
V_{0} & \text { otherwise }
\end{array}\right. \\
& V(\rho)= \begin{cases}0 & \rho \leq R \\
V_{0} & \text { otherwise }\end{cases} \\
& V(r)=\left\{\begin{array}{cc}
0 & r \leq R \\
V_{0} & \text { otherwise }
\end{array}\right.
\end{aligned}
$$

For a GaAs $/ \mathrm{Al}_{\mathrm{x}} \mathrm{Ga}_{1-\mathrm{x}} \mathrm{As}$ interface, the barrier height $V_{0}$ is taken to be $85 \%$ of the total energy-band-gap difference between the two semiconductors: $\Delta E_{g}=1.155 x+0.37 x^{2} \mathrm{eV}$ [22]. Here $V_{0}=101.3 \mathrm{meV}$, when $x=0.1$.

The units of length and energy used throughout are the polaron radius $l=\left(\frac{\hbar}{2 m * \omega}\right)^{1 / 2}$ and the polaron energy $\hbar \omega$ where $\omega$ is the phonon frequency, and $m *$ is the effective mass of GaAs. With these units, the Eq. (1) becomes

$$
H=-\left[\frac{\partial^{2}}{\partial \rho^{2}}+\frac{1}{\rho} \frac{\partial}{\partial \rho}+\frac{1}{\rho^{2}} \frac{\partial^{2}}{\partial \varphi^{2}}\right]-\frac{\chi}{\sqrt{\rho^{2}+z^{2}}}+\frac{V_{w}(z)}{R^{*}}
$$

The parameter, $\chi=\frac{e^{2}}{\hbar \varepsilon_{\infty}}\left(\frac{2 m^{*}}{\hbar \omega}\right)^{1 / 2}$ where $\varepsilon_{\infty}$ is the optic dielectric constant. In the absence of electron-phonon interaction $\varepsilon_{\infty}$ is replaced by $\varepsilon$. Since an exact solution of the Schrödinger equation with the Hamiltonian in Eq. (4) is not possible, a variational approach has been adopted. The wave functions of the ground state donor impurity for these systems are 


$$
\begin{aligned}
& \psi_{Q W}=\left\{\begin{array}{cc}
N_{1} \cos \left(k_{1} z\right) e^{-\alpha r} & |z| \leq \frac{L}{2} \\
N_{2} e^{k_{2} z} e^{-\alpha r} & \text { otherwise }
\end{array}\right. \text { for a quantum well, } \\
& \psi_{Q W W}=\left\{\begin{array}{ll}
N_{3} \cos \left(k_{1} \rho\right) e^{-\beta r} & \rho \leq R \\
N_{4} e^{k_{2} \rho} e^{-\beta r} & \text { otherwise }
\end{array}\right. \text { for a quantum well wire and } \\
& \psi_{Q D}=\left\{\begin{array}{cc}
N_{5} \sin \left(\frac{k_{1} r}{r}\right) e^{-\delta r} & r \leq R \quad \text { for a quantum dot } \\
N_{6} e^{k_{2} r} e^{-\delta r} & \text { otherwise }
\end{array}\right.
\end{aligned}
$$

where $k_{1}=\sqrt{2 m^{*} E_{s} / \hbar^{2}}, k_{2}=\sqrt{2 m^{*}\left(V-E_{s}\right) / \hbar^{2}}, E_{s}$ is the sub-band energy. Here $N_{1}, N_{2}$, $N_{3}, N_{4}, N_{5}$ and $N_{6}$ are normalization constants and $\alpha, \beta$ and $\delta$ are the variational parameters. By matching the wave functions and their derivatives at boundaries of the well and along with the normalization, we fix all the constants except the variational parameters.

The Schrödinger equation is solved variationally by finding $\langle H\rangle_{\min }$ and the binding energy of the donor in a quantum well is given by the difference between the energy with and without Coulomb term.

The binding energy of ground state with phonons is given by

$$
E_{b}^{p h}=E_{s}^{p h}-\langle H\rangle_{\min },
$$

where $\quad E_{s}^{p h}=\left\langle\psi_{\text {sub }}\left|-\nabla^{2}+V-\frac{\pi}{2} \tau+\frac{\pi}{4} \tau \nabla^{2}\right| \psi_{\text {sub }}\right\rangle \quad$ with $\quad \tau=\frac{1}{2}\left(\frac{1}{\varepsilon_{\infty}}-\frac{1}{\varepsilon}\right)\left(\frac{e^{2}}{\hbar \omega l}\right) \quad$ and $\varepsilon\left(\varepsilon_{\infty}\right)$ are the static and high frequency dielectric constants of GaAs. We follow the same procedure for calculating the binding energy with phonons as done in the Ref. [9] wherein they investigated the effect of electron-phonon interaction for the QWs whereas we have extended the same for the QWWs and QDs.

\section{Results and Discussion}

Numerical calculations have been carried out to investigate the polaron binding energy of the donor with the well size. We have calculated the effect of the electron-bulk phonon interaction on the binding energy for the on-center impurity. For numerical calculations, we have chosen the $\mathrm{GaAs} / \mathrm{Al}_{\mathrm{x}} \mathrm{Ga}_{1-\mathrm{x}} \mathrm{As}$ as a superlattice, since this system is the well known and almost all the properties are known. We have chosen $\mathrm{GaAs}$ and $\mathrm{Al}_{\mathrm{x}} \mathrm{Ga}_{1-\mathrm{x}} \mathrm{As}$ because of the same crystal structures and lattice constants [23]. The parameters pertaining to the system are: $m^{*}=0.067 m_{0}, \varepsilon=13.13, \varepsilon_{\infty}=10.89$ and $\hbar \omega=36 \mathrm{meV}$.

Fig. 1 shows the variation of binding energy with the well width with and without the inclusion of phonons. We observe that impurity binding energy, with and without the 
inclusion of phonons, decreases as the well size increases. As an increase in the well size resulting a spreading of the wave function consequently causes lowering of binding

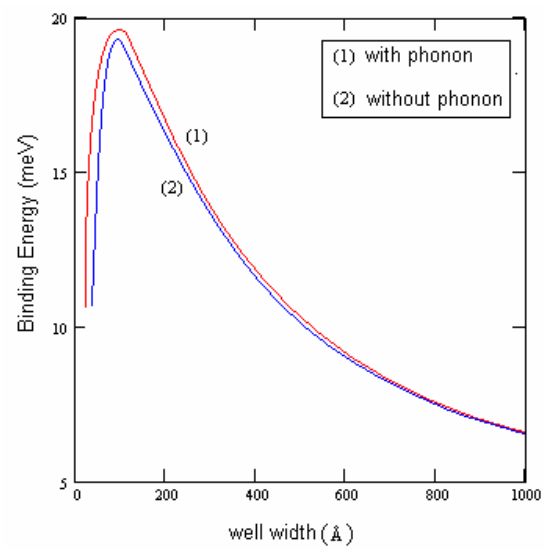

Fig.1. Variation of binding energy with the well width with and without the inclusion of phonons. The curve 1(2) corresponds to the case with (without) electron-bulk phonon coupling.

energy. While the binding energy increases with a decrease of well size, reaching a maximum value and then decreases when the well size still decreases. As the well size approaches zero the confinement becomes negligibly small, and in the finite barrier problem the tunneling becomes huge. The binding energy again approaches the bulk value of the barrier. In both the cases, the binding energy approaches the bulk value in both the limits of $L \rightarrow 0$ and $L \rightarrow \infty$ corresponding to $5.3 \mathrm{meV}$ which is value of binding energy of GaAs in a bulk limit.

It is implied that the polaronic effect on the binding energy of the impurity is defined as difference between the binding energy in the presence and absence of phonons. This has been explicitly demonstrated in this figure. So also we have observed that there is an increase of binding energy when electron-phonon interaction is included in the Hamiltonian. This increase in binding energy with inclusion of electron-phonon interaction is good agreement with the Ref. [9]. Moreover, the results demonstrate that the correction with respect to the bulk-phonon LO on the binding energies is higher for small wells than for large wells. The observed increase in binding energy is around 1 $\mathrm{meV}$ for $100 \AA$. But this difference in binding energy becomes smaller when the well size becomes larger and larger.

Fig. 2 displays the variation of binding energy with the wire radius with and without the inclusion of electron-phonon interaction. The feature of binding energy in QWW is the same as that of the behavior of donor binding energy in the QW. But it is observed that there is an increase of binding energy in QWW due to the confinement. It has been observed that the binding energies in QWW reach values in the range of 2.5-3.5 times the bulk Rydberg [14] whereas we are able to obtain the donor binding energies 1.5 times the bulk Rydberg for $100 \AA$. Moreover, this effect is more pronounced for thin wires. The increase of observed polaron energy is around $1.5 \mathrm{meV}$ for $100 \AA$. 




Fig.2. Variation of binding energy with the wire radius with and without the inclusion of phonons. The curve 1(2) corresponds to the case with (without) electron-bulk phonon coupling.

We present the variation of binding energy with the dot radius with and without the inclusion of phonons in Fig.3 for QDs. We observe the same features which have observed for the QWs and QWWs. In quantum dots, it is more difficult to obtain accurate values for the exciton polaron energies from spectroscopic data [24]. But it is observed that there is still increase of binding energy in QDs due to the confinement. The increase in polaron energy is around $5 \mathrm{meV}$ for $100 \AA$. We present the variation of polaron energy with the dimension with the inclusion of phonons for three different confinements in Fig.4. The binding energy with the electron-phonon interaction has the maximum energy for QDs due to the confinement. And this effect is more pronounced when size of QWs, QWWs and QDs becomes smaller and smaller.



Fig.3. Variation of binding energy with the dot radius with and without the inclusion of phonons. The curve 1(2) corresponds to the case with (without) electron-bulk phonon coupling. 




Fig. 4. Variation of binding energy with the dimension with the inclusion of phonons for three different confinements. The curves 1, 2, and 3 correspond to the cases of QD, QWW and QW.

\section{Summary}

The effect of bulk Longitudinal-Optical phonon on donor binding energy of a hydrogenic donor impurity is investigated for a GaAs$/ \mathrm{Al}_{\mathrm{x}} \mathrm{Ga}_{1-\mathrm{x}} \mathrm{As}$ system. The calculations have been carried out for the low-dimensional semiconducting systems like quantum well, quantum well wire and quantum dot using a variational approach within the single band effective mass approximation. The results show that the polaronic effect is more prominent for the lowest confinement. The polaronic effect enhances the donor binding energy but it diminishes when the well width, wire radius and dot radius become larger. Also the numerical calculations reveal that the effect of electron-phonon interaction on the donor binding energy are considerable and should not be neglected especially for narrow dimensions in all the three confinements. Experimental efforts are expectant to provide support to our calculations especially for quantum dots.

\section{References}

1. Ferreira da Silva, Phys. Rev. B 41, 1684 (1990). doi:10.1103/PhysRevB.41.1684

2. A. Montes, C. A. Duque, and N. Porras-Montenegro, J. Phys.: Cond. Matt. 10, 5351 (1998). doi:10.1088/0953-8984/10/24/012

3. B. S. Monozon, J. L. Dunn, and C. A. Bates, Phys. Stat. Solidi B 210, 661 (1998). doi:10.1002/(SICI)1521-3951(199812)210:2<661::AID-PSSB661>3.0.CO;2-P

4. S. L. Ban and J. E. Hasbun, Phys. Rev. B 59, 2276 (1999). doi:10.1103/PhysRevB.59.2276

5. Wang Rui-Qiang, Xie Hong-Jing, Guo Kang-Xian, Yu You-Bin, and Deng Yong-Qing, Commun. Theor. Phys. 43, 169 (2005). doi:10.1088/0253-6102/43/1/032

6. Hong-jing Xie, Chuan-yu Chen, and Ben-kun Ma, J. Phys.: Cond. Matt. 12, 8623 (2000) ibid Phys. Rev. B 61, 4827 (2000).

7. K. Sellami and S. Jaziri, Physica E 26, 143 (2005). doi:10.1016/j.physe.2004.08.041

8. K-X. Guo and C.-Y. Chen, Tr. J. Phys. 21, 1261 (1997).

9. L. Tayebi, M. Fliyou, Y. Boughaleb and L. Bouziaene, M. J. Condensed Matter 5, 128 (2004). 
10. M. H. Degani and O. Hipolito, Phys. Rev. B 35, 4507 (1987). doi:10.1103/PhysRevB.35.4507

11. U. Bockelmann and G. Bastard, Phys. Rev. B 42, 8947 (1990). doi:10.1103/PhysRevB.42.8947

12. W. Weigscheider, L. N. Pfeiffer, M. M. Dignum, A. Pinczuk, K. W. West, S. L. McCall, and R. Hull, Phys. Rev. Lett. 71, (1993) 4071. doi:10.1103/PhysRevLett.71.4071

13. M. Bouhassoune, R. Charrour, M. Fliyou, D. Bria, and A. Nougaoui, J. Appl. Phys. 91, 232 (2002). doi:10.1063/1.1419261

14. R. C. Ashoori, H. L. Stormer, J. S. Weiner, L. N. Pfeiffer, K. W. Baldwin, and K. W. West, Phys. Rev. Lett. 71, 613 (1993). doi:10.1103/PhysRevLett.71.613

15. P. L. McEuen, E. B. Foxman, U. Meirav, M. A. Kastner, Y. Meir, and N. S. Wingreen, Phys. Rev. Lett. 66, 1926 (1991). doi:10.1103/PhysRevLett.66.1926

16. J. Weis, R. J. Haug, K. V. Klitzing, and K. Ploog, Phys. Rev. Lett. 71, 4019 (1993). doi:10.1103/PhysRevLett.71.4019

17. B. Meuer, D. Heitmann, and K. Ploog, Phys. Rev. Lett. 8, 1371 (1992). doi:10.1103/PhysRevLett.68.1371

18. C. M. Lee and S.W. Gu, Sol. Stat. Commun. 116, 51 (2000). doi:10.1016/S0038-1098(00)00279-9

19. I. P. Ipatova, A. Yu. Maslov, and O. V. Proshina, Europhys. Lett. 53 (6), 769 (2001). doi:10.1209/epl/i2001-00218-2

20. V. S. Bagaev, V. V. Zaïtsev, E. E. Onishchenko, and Yu.G. Sadoyev, J. Cryst. Growth 214, 250 (2000). doi:10.1016/S0022-0248(00)00079-8

21. J. T. Devreese, in Encyclopedia of Applied Physics VCH, Weinheim, 1996, p. 383, Vol. 14.

22. H. J. Lee, L. Y. Juravel, J. C. Wolley, and A. J. Springthorpe, Phys. Rev. B 21, 659 (1980). doi:10.1103/PhysRevB.21.659

23. M. Tanaka and Y. Higo, Phys. Rev. Lett. 87, 026602 (2001). doi:10.1103/PhysRevLett.87.026602

24. Y. Nagamune, M. Nishioka, S. Tsukamoto, and Y. Arakawa, Appl. Phys. Lett. 64, 2495 (1994). doi:10.1063/1.111577 